\title{
Nutritional Requirements in Geriatrics
}

\author{
Anne Marie Beck and Mette Holst
}

\section{Abstract}

The purpose of this chapter is to describe the nutritional recommendations for older adults and change in requirements with age and disease. Key factors influencing nutritional requirements, dietary intake, and nutritional status in old adults will be described, including specific nuances for geriatric and orthogeriatric patients.

\section{Keywords}

Nutritional recommendations $\cdot$ Nutritional status $\cdot$ Nutritional risk factors

This chapter is a component of Section 1: Nutritional Care in Old Age.

For an explanatiocn of the grouping of chapters in this book, please see Chap. 1: "Overview of Nutrition Care in Geriatrics and Orthogeriatrics."

\footnotetext{
A. M. Beck ( $\bowtie)$

University College Copenhagen, Institute of Nursing and Nutrition, Copenhagen, Denmark

Dietetic and Nutritional Research Unit, Herlev-Gentofte University Hospital,

Herlev, Denmark

e-mail: ambe@kp.dk

M. Holst

Department of Clinical Medicine, Centre for Nutrition and Bowel Disease, Aalborg

University Hospital, Aalborg University, Aalborg, Denmark

e-mail: mette.holst@rn.dk
}

Ó. G. Geirsdóttir, J. J. Bell (eds.), Interdisciplinary Nutritional Management and 


\section{Learning Outcomes}

By the end of this chapter, you will be able to:

- Report common nutritional risk factors among different groups of older adults.

- List examples of nutritional recommendations for old adults in relation to energy, protein, and vitamin D.

- Justify changes in requirements with age and diseases.

- Identify potentially modifiable risk factors for malnutrition in older adults.

- Describe aging-related characteristics that may impede accurate dietary assessment.

\subsection{Nutritional Recommendations for Older Adults, Geriatric and Orthogeriatric Patients}

This chapter will describe the nutritional recommendations for older adults focusing on where the recommendations differ from the requirement for younger people [15]. Many geriatric guidelines about food and nutrition are primarily valid for groups of healthy individuals with various levels of physical activity [2-4]. For individuals with diseases and other groups with special needs, dietary recommendations and the consequent dietary composition and energy content might have to be adjusted accordingly [1]. This section will also describe how nutritional requirements change in relation to aging and disease processes, with a specific focus on energy and protein and vitamin D.

\subsection{Nutritional Recommendations for Older Adults}

Dietary reference values (DRVs) for essential nutrients include the average requirement (AR), recommended intake (RI), upper intake level (UL), lower intake level (LI), and reference values for energy [2]. It is important to distinguish between the average requirement for a nutrient and the recommended intake of a nutrient. The recommended intake represents more than the requirement for the average person and covers the individual variations in the requirement for the vast majority of the population group (Fig. 2.1). Depending on the criteria used for setting the average requirement, the safety margin between the average requirement and recommended intake can vary [2]. In general, aging does not change the DRVs except for energy, protein, and vitamin D. However, it should be noted that low energy intake is recognized as a risk factor for macro- and micronutrient deficiency [2].

\subsubsection{Energy Requirement and Recommended Intake}

Daily energy expenditure tends to decline with age in the absence of disease mainly due to decreased fat-free mass (FFM) and decreased physical activity levels [2]. There are two main approaches able to be applied by interdisciplinary healthcare 
Fig. 2.1 Frequency distribution of an individual nutrient requirement. $S D$ standard deviation. (With permission from [2]. https://www.norden.org/en/ publication/ nordic-nutrition-recommen dations-2012)

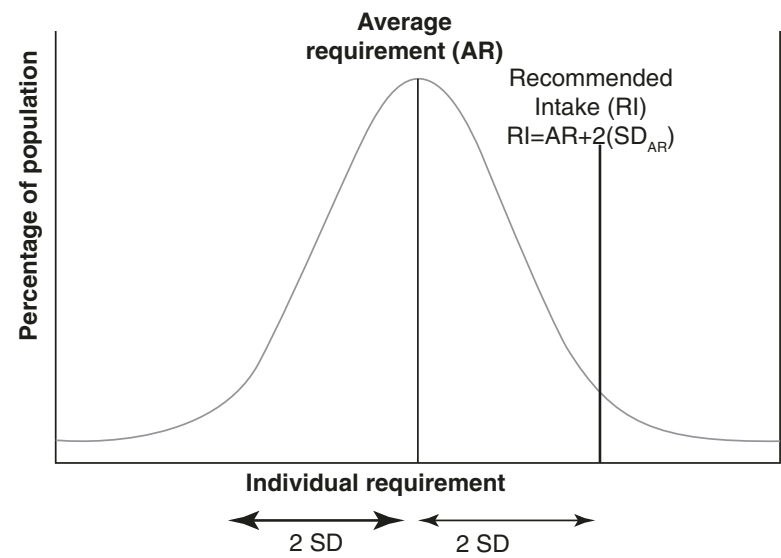

providers in the clinical setting to estimate energy requirements. The factorial method calculates total energy expenditure from the resting (or basal) energy expenditure multiplied by a factor indicating physical activity level (PAL) [6]. An alternate method is the ratio method, which is an even more pragmatic approach whereby the subject's weight is multiplied by a specified caloric or kilojoule amount [6]. In the latter approach, the use of an adjusted body weight might be considered for older people who are obese. For older people with, e.g., a low body mass index, where weight gain might be relevant, the energy cost of weight gain may also be included. In these cases, one should be aware of the risk of refeeding (Chap. 3). While the factorial and ratio methods are broadly applied international in clinical settings, which method should be applied and what values to use are perhaps equally related to local practices and evidence-based values [2, 7-9]. Because estimates of requirements vary across different populations and clinical guidelines $[1,8,9]$, we would recommend looking to country- or disease-specific recommendations. A useful good starting point is the E.S.P.E.N. guidelines for geriatrics which can be found here: https://www.espen.org/guidelines-home/espen-guidelines.

In this guideline about $30 \mathrm{kcal} / \mathrm{kg} \mathrm{BW}$ is suggested as a rough estimate and general orientation for energy requirements in older persons. This guiding value needs individual adjustment regarding all relevant factors, i.e., gender, nutritional status, physical activity, and clinical condition. In addition, the aim of nutritional support (e.g., weight maintenance or increase) and acceptance and tolerance of the nutritional intervention need to be considered [1]. Because of great heterogeneity and large individual variation of energy requirements, even in healthy older persons, adequacy of energy intake needs to be controlled by close monitoring of body weight (taking water retention or losses into account), and intake adapted accordingly. "Injury factors" are applied in various settings for either the factorial method or the ratio method. These are generally not strongly evidenced, although consensus documents exist to support consideration of the increased requirements of acute or chronic disease [10]. Again, it should be noted that these are only a starting point and should include ranges rather than exact figures. Close monitoring should be prioritized above reliance on initial estimates, particularly in individuals considered likely to have particularly high requirements. It should be kept in mind that 
spontaneous oral energy intake of acutely hospitalized older patients is usually low and does not cover requirements [1]. Where accurate individual measures are necessary, alternative approaches, for example, indirect calorimetry or even doubly labeled water (DLW) technique in which stable isotopes ( $2 \mathrm{H}$ and $18 \mathrm{O})$ are administered orally, could be considered, although these are primarily used for research.

\subsubsection{Protein Requirement and Recommended Intake}

Multimorbidity and chronic diseases are more frequent in older adults, and such conditions might lead to periodic temporary losses of body protein through catabolic exacerbations of the disease, temporary periods of bed rest, or loss of appetite. The losses must be replaced from the diet and thus represent an added need for dietary protein compared to the traditional recommendation defined as the lowest intake of protein to maintain nitrogen balance. In some cases positive nitrogen balance may not be achieved regardless of the amount of protein provided [2]. In addition, older individuals exhibit a gradual loss of muscle mass and strength with age (sarcopenia, Chap. 8). Sarcopenia may also occur in a variety of other nonnutritive factors, for example, in a sedated intensive care patients with induced paralysis, who will also lose muscle despite adequate protein and energy intakes [2].

A suggested minimum protein intake for many older adults across settings is 1.0-1.2 g protein/kg body weight (BW)/day. However, a systematic review suggested that a safe intake of up to at least $1.2-1.5 \mathrm{~g}$ protein $/ \mathrm{kg} \mathrm{BW} /$ day or approximately 15-20 E\% represents an optimal intake level for older adults [2]. With decreasing energy intake (below $8 \mathrm{MJ} / \mathrm{d}$ ), the protein $\mathrm{E} \%$ should be increased accordingly $[1,2]$. These recommendations are intended for the general population and not for groups or individuals with diseases or other conditions that affect their nutrient requirements. They are meant to be used for prevention purposes and are not specifically meant for treatment of diseases or significant weight reduction. Recommendations generally cover temporarily increased requirements, for example, during short-term mild infections or certain medical treatments. The recommended amounts are usually not suited for long-term infections, malabsorption, or various metabolic disturbances or for the treatment of persons with a nonoptimal nutritional status $[1,2]$.

In case of illness, protein requirements may even be further increased, e.g., due to inflammation, infections, and wounds. Very little is known about the protein needs of frail and multimorbid older persons, and scientific evidence, e.g., from intervention trials, is presently insufficient to derive concrete figures. Consequently, until more evidence is available, an intake of at least $1.0 \mathrm{~g} / \mathrm{kg} \mathrm{BW} /$ day should be ensured in all older persons, particularly in those at risk of malnutrition, e.g., frail and multimorbid persons, whose intake is often far below this amount [1]. Increased requirements, e.g., for muscle growth with strength training, for tissue regeneration in malnutrition or wound healing, or for increased metabolic demands in case of critical illness, should be met by appropriately increased intake [1]. Daily amounts 
of $1.2-1.5 \mathrm{~g} / \mathrm{kg}$ BW/day have been suggested for older persons with acute or chronic illness and up to $2.0 \mathrm{~g} / \mathrm{kg} \mathrm{BW} /$ day in case of severe illness, injury, or malnutrition [1]. It is important to bear in mind that an insufficient intake of energy increases protein requirement. Thus, regarding protein status it is important to ensure not only adequate intake of protein but also appropriate intake of energy [1].

It is also worth noting that protein status is hard to estimate in daily clinical practice or community settings. Nitrogen balance remains the method of choice for determining the protein requirement in adults in the absence of validated or accepted alternatives and in the absence of a reliable biological marker of protein status [2]. However, N-balance is primarily measured for research purpose. Serum biomarkers as serum albumin and serum prealbumin or other biomarkers have not been shown to be valid method to estimate protein status [1].

\subsubsection{Micronutrients and Dietary Fibers}

With low energy intakes, it is difficult to meet the needs for all nutrients. In such cases supplementation with a multivitamin/mineral tablet and, where fiber intake appears inadequate or symptoms are suggestive of inadequacy, dietary fiber should be considered. For groups with a very low energy intake $(<6.5 \mathrm{MJ}$ or $1550 \mathrm{kcal})$, supplementation with a multivitamin/mineral tablet is recommended [1, 2].

Micronutrient recommendations vary according to populations, comorbidities, and national recommendations and clinical guidelines [5]. We recommend referral to national recommendations in the absence of disease (see some suggestions for further reading at the end of the chapter). Where multiple comorbidities exist and there is expected deficiency or deficiencies, a dietitian or medical nutrition specialist referral should be considered.

While a broad array of micronutrient deficiencies will be encountered by clinicians working with older adults, one that is most likely to be routinely identified is vitamin D. Vitamin D requirement is higher in older adults than in younger people. Apart from low dietary intake and limited time spent outdoors, the reason is that the amount of 7-dehydrocholesterol in the skin epidermis diminishes with age and the efficiency of conversion of this precursor into vitamin D is less effective than in younger individuals [2]. There is also some evidence that the PTH concentration tends to be higher among the older adults compared to younger adults at similar serum 25OHD concentrations. ${ }^{1}$ This might indicate less efficient bioconversion due to diminished kidney function resulting in secondary hyperparathyroidism [2]. Further, the more rapid bone loss and higher fracture rate in older women than in men is related to diminished estrogen production in postmenopausal women [2]. More details about vitamin D recommendations, bone health, and osteoporosis can be found in Chap. 9.

\footnotetext{
${ }^{1}$ The circulating serum $25 \mathrm{OHD}$ concentration is regarded as a good marker of vitamin D status (2).
} 


\subsection{Nutritional Risk Factors in Older Adults}

As shown in Fig. 2.2, multiple factors have been correlated with malnutrition in older adults. Nutritional risk factors include reduced appetite, female sex, social resources, poor physical function, poor self-related health, sensory function, chewing and swallowing problems, physical and cognitive impairment, and many other factors affecting the social, mental, and physical health of the old adult [11]. Chewing and swallowing problems due to dysphagia will be discussed in detail in Chap. 18.

Less emphasis has focused on nutritional risk factors that could be considered potentially modifiable. This was therefore the focus in a recent systematic review performed as part of the MaNuEL program [12]. Prospective cohort studies with

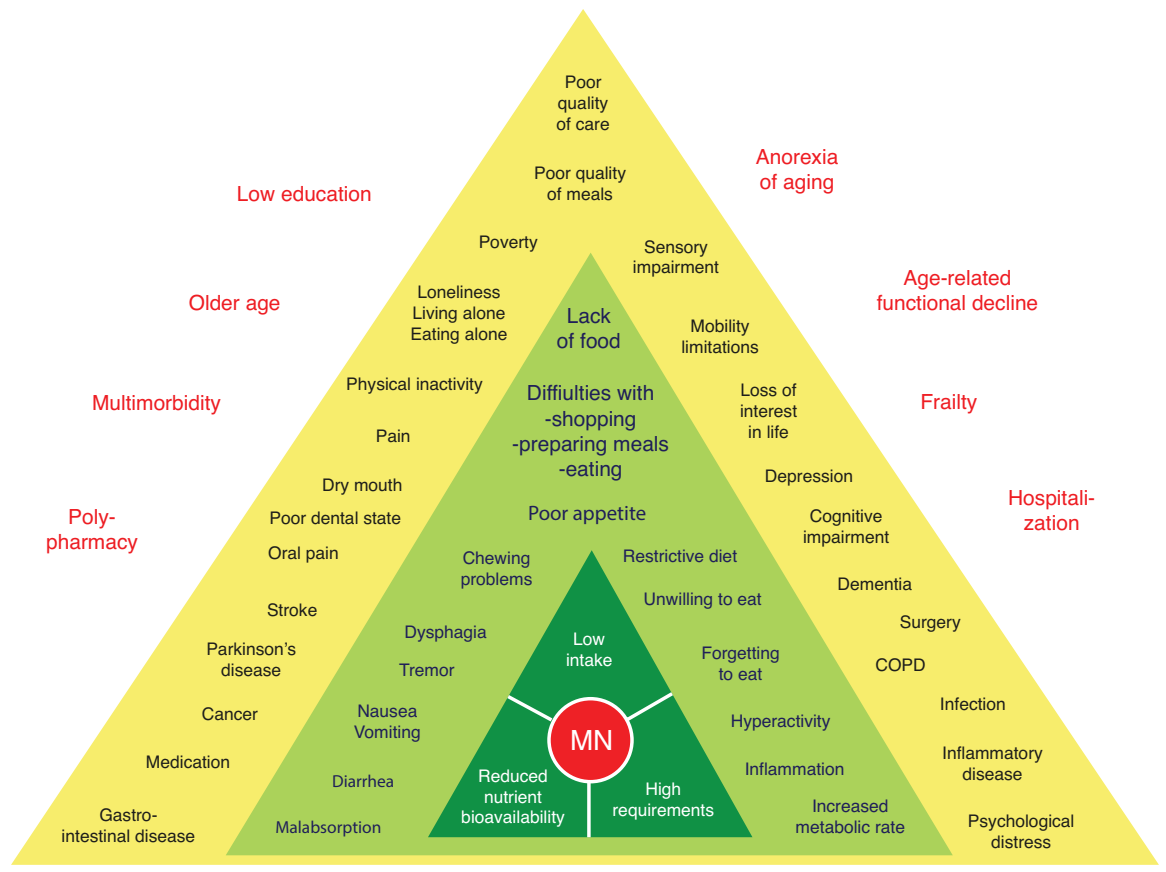

Fig. 2.2 DoMAP model. All factors-independent of the level-are regarded as (potential) "determinants" of MN meaning that they may contribute to the development of MN in a causative manner. The levels illustrate different modes of action: Level I (dark green): Central etiologic mechanisms; Level 2 (light green): Factors in this level directly lead to one of the three mechanisms in Level I (e.g., swallowing problems may directly cause low intake): Level 3 (yellow): Factors in this level may indirectly lead to one (or more) of the three central mechanisms through one (or more) of the direct factors in the light green triangle (e.g., stroke may cause low intake via dysphagia or difficulties with eating); surrounding factors in red are age-related changes and general aspects which also contribute to the development of $\mathrm{MN}$, but act even more indirectly or subtle. DoMAP Determinants of Malnutrition in Aged Persons, $M N$ malnutrition 
participants of a mean age of 65 years or over in all settings were included. Malnutrition was defined as, e.g., low BMI or weight loss or as assessed by a screening tool [11]. The results are summarized in Table 2.1.

One, if not the most important, determinant of dietary intake in older persons is appetite which declines with age, referred to as anorexia of aging. The reported prevalence of anorexia of aging ranges from up to $25 \%$ in home dwellers to $62 \%$ in hospital populations and $85 \%$ in nursing home populations [13]. The consequences of anorexia of aging include the development of subsequent malnutrition, immunosuppression, sarcopenia, and frailty, which can reciprocally worsen appetite further [14]. This ultimately leads to adverse outcomes with higher rates of morbidity and mortality. The causes of anorexia of aging include changes in peripheral hormone signaling, gut motility, and sensory perception due to aging as well as social and environmental factors [13]. Regrettably, until now a uniform, unambiguous definition of anorexia of aging is lacking [15]. Screening tools for appetite assessment exist, for example, AHSPQ (Appetite, Hunger and Sensory Perception Questionnaire) or SNAQ (Simplified Nutritional Appetite Questionnaire); they are however not well established [15]. Further, in a recent systematic review, the authors concluded that there was a lack of clarity about whether anorexia of aging or malnutrition was the intervention target and that there was a need for standardized assessment so that effectiveness of a range of interventions could be fully explored [13].

Older adults have an increased risk at an insufficient protein intake because preferential consumption of protein-rich foods may decrease with aging [16]. Knowledge about the prevalence of low protein intake across different settings is limited. However, a meta-analysis applying data from four cohorts and four national surveys showed that the overall pooled prevalence of protein intake below recommended was up to $70 \%$ [16].

Table 2.1 Potentially modifiable nutritional risk factors for malnutrition in older adults (based on results from O'Keeffe et al. 2019 [11])

\begin{tabular}{|c|c|c|}
\hline Risk factors & $\begin{array}{l}\text { Determinants of } \\
\text { malnutrition }\end{array}$ & $\begin{array}{l}\text { Level of } \\
\text { evidence }\end{array}$ \\
\hline $\begin{array}{l}\text { Hospitalization, eating dependency, poor self-perceived health, } \\
\text { poor physical function, and poor appetite }\end{array}$ & Yes & Moderate \\
\hline $\begin{array}{l}\text { Loss of interest in life, access to meals and wheels, and } \\
\text { modified texture diets }\end{array}$ & Yes & Low \\
\hline $\begin{array}{l}\text { Chewing difficulties, mouth pain, gum issues comorbidity, } \\
\text { visual and hearing impairments, smoking status, alcohol } \\
\text { consumption and physical activity levels, complaints about } \\
\text { taste of food and specific nutrient intake }\end{array}$ & No & Moderate \\
\hline $\begin{array}{l}\text { Psychological distress, anxiety, loneliness, access to transport } \\
\text { and well-being, hunger, and thirst }\end{array}$ & No & Low \\
\hline $\begin{array}{l}\text { Dental status, swallowing, cognitive function, depression, } \\
\text { residential status, medication intake and/or polypharmacy, } \\
\text { constipation, periodontal disease }\end{array}$ & $\begin{array}{l}\text { Conflicting } \\
\text { results }\end{array}$ & - \\
\hline
\end{tabular}




\subsection{Estimating Intake in Older Adults}

Assessment of dietary intake of older people requires particular care (Table 2.2). Diminished functions, disabilities, and health disorders may impair the ability to recall or record dietary intake correctly. In large epidemiological studies with older persons, generally the same dietary assessment methods as for younger age groups are applied-mainly food frequency questionnaires, diet histories, and multiple 24-h recalls - which all have their strengths and limitations regardless of age. It seems like these usual methods for dietary assessment are valid for older persons if they are physically and mentally healthy [15]. In clinical settings, food and hydration recording are most used method and can give good insight of food intake if healthcare providers, and if possible patient or family/friends, are involved in the process of food recording [15]. With increasing impairments, frailty, and disabilities, more time and effort should be invested in assessment of dietary intake needs among old adults.

These age-related issues are especially prevalent among old adults in shortor long-term institutional care, for example, hospitals and residential aged care homes. In these settings, direct observation, and direct measurement of dietary intake, is possible and should be used instead of or to assist self-reporting methods. Assistance from caregivers may be available, and information on food preparation, recipes, and usual portion sizes can be obtained from the kitchen personnel [15]. The gold standard in this setting is to weigh all meal components on the plate before and after meals, which may be a challenging task when snacks, in-between meals, and beverages also must be included. This method is extremely time-consuming and laborious and is generally reserved for research restricted to small patient groups [15]. Estimation methods are much more suitable regarding time and effort, and in clinical practice in geriatric institutions, plate diagrams with estimation of intake in quarters of the offered meal are widely used (Fig. 2.3) [15, 17, 18].

Table 2.2 Age-related characteristics that may impede accurate dietary assessment (based on Volkert and Schrader 2013 [14])

- Reduced capacity to deal with stress

- Physical limitations

- Visual impairment

- Hearing problems

- Difficulties writing

- Mental impairment

- Reduced short-term memory

- Cognitive decline, dementia

- Reduced communication skills: Limited attention, divagation

- Persons may not or only little be involved in food preparation 


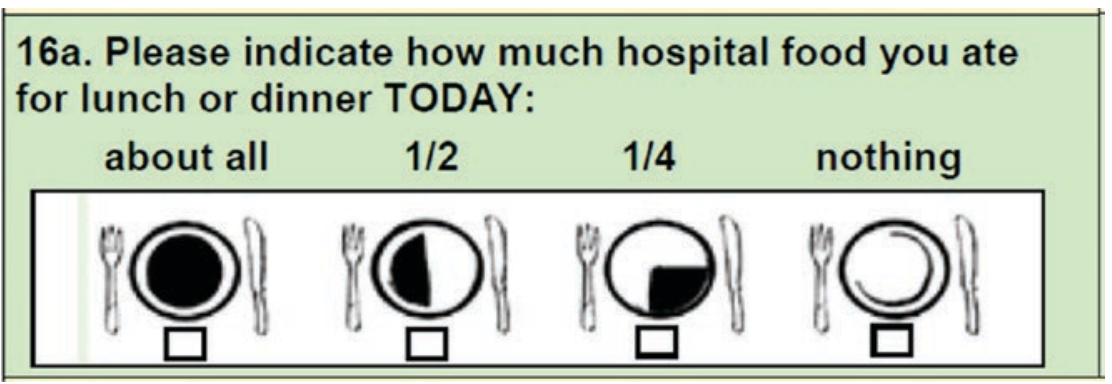

Fig. 2.3 Example of a simple estimation method to assess dietary intake, which can be used by the patients (from the Nutrition Day survey in hospitals [18] [https://www.nutritionday.org/])

\subsection{Nutritional Status of Older Adults, Geriatric and Orthogeriatric Patients}

Malnutrition, according to WHO definition, includes undernutrition (wasting, stunting, underweight), inadequate vitamins or minerals, overweight, obesity, and resulting diet-related noncommunicable diseases [19]. Overweight and obesity in old adults is described in Chap. 15. Malnutrition can be defined as "a state resulting from lack of intake or uptake of nutrition that leads to altered body composition (decreased fat free mass) and body cell mass leading to diminished physical and mental function and impaired clinical outcome from disease" [20]. Malnutrition can result from starvation, disease, or advanced aging (e.g., $>80$ years), alone or in combination [20]. Although there is no gold standard diagnosis for malnutrition, global consensus around core diagnostic criteria for malnutrition in adults in clinical settings is emerging [19]. With two-step approach for the malnutrition diagnosis, i.e., first screening to identify "at-risk" status using any validated screening tool and, second, assessment for diagnosis and grading the severity of malnutrition [21]. Other sections of this book detail nutritional assessment, nutritional diagnosis, and treatment (Chap. 3); malnutritional prevention (Chap. 4); nutritional support (Chap. 5); the overlap between co-diagnoses of malnutrition, sarcopenia, cachexia, and frailty (Chap. 8); and why systematized, interdisciplinary nutritional care is key to high-value, patient-centered outcomes (Chaps. 1 and 13).

As can be seen from Fig. 2.4, reported malnutrition prevalence rates vary considerably between and even within the settings. This might be explained by differences in age, morbidity, and functional status of included participants in the studies. Still, the results confirm that malnutrition prevalence markedly increases from community dwellers to geriatric and orthogeriatric patients and even further to residents in 


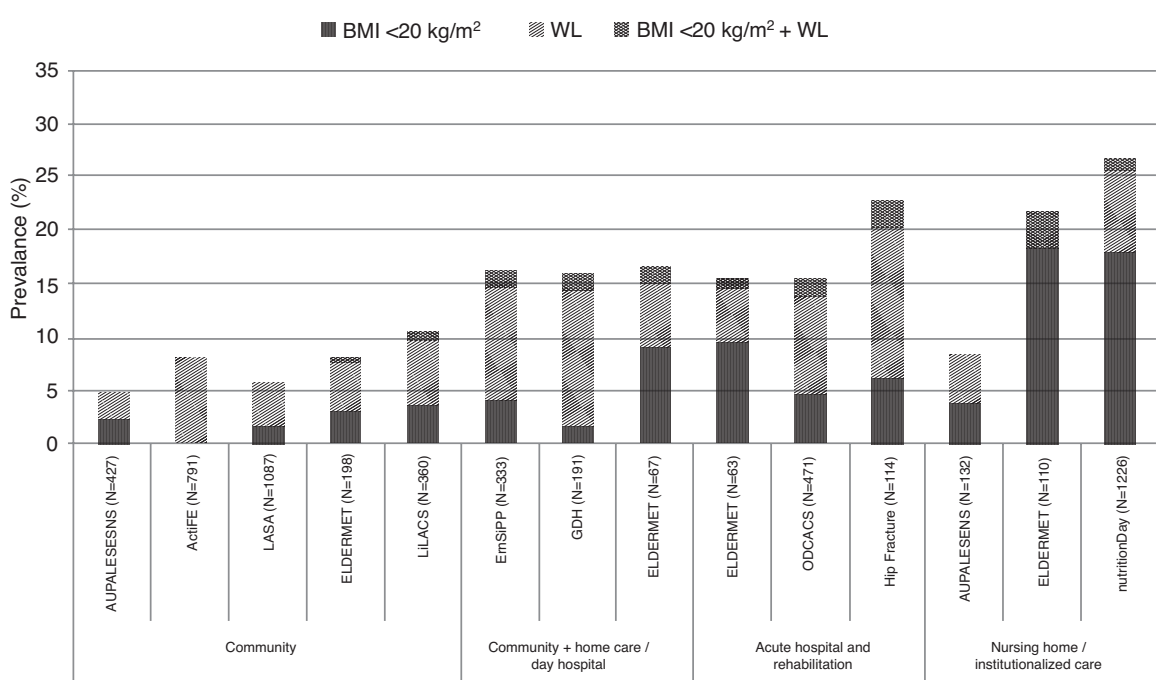

Fig. 2.4 Prevalences of malnutrition criteria based on low BMI, weight loss (WL) or both in older adults in different study samples by setting. (With permission from: Wolters M, Volkert D, Streicher M, Kiesswetter E, Torbahn G, O'Connor EM, et al. Prevalence of malnutrition using harmonized definitions in older adults from different settings - A MaNuEL Study. Clin Nutr. 2019;38(5):2389-98 [22])

nursing homes [22]. These findings are supported by the findings of reduced intake of energy during disease, due to among a high prevalence of nutritional risk factors. The complexity of malnutrition and nutritional risk factors in multimorbid old adults highlight the importance of interdisciplinary and multimodal interventions as also recommended by the E.S.P.E.N. guideline for geriatric patients [1]. These findings also highlight the need to proactively prioritize screening and interventional processes in the community settings (Chaps. 4 and 13).

\subsection{Summary}

Deterioration of nutritional status in older adults is commonly observed and is associated with impairment of recovery and rehabilitation. In older adults this can occur rapidly during acute illness or insidiously across time in response to diverse age or chronic disease-related factors. Insufficient energy and protein intake is a common cause of poor nutritional status in old age and specially during hospitalization. Older persons are generally at risk of malnutrition due to anorexia of aging but also several nutritional risk factors of which several might be modifiable. We conclude that malnutrition in older adults is a complex condition; interdisciplinary teamwork is needed to support and sustain a healthy nutritional status throughout the aging years. 


\section{Take-Home Points}

- Impaired nutritional status is frequent among geriatric and orthogeriatric patients.

- Insufficient intake of energy, protein, and vitamin D is a significant problem among older adults, geriatric and orthogeriatric patients.

- Potentially modifiable nutritional risk factors should be taken into consideration when planning nutritional interventions.

- Ongoing monitoring should confirm whether the estimates of requirements (and intake) maintain or improve nutritional status over time.

\section{References}

1. Volkert D et al (2019) ESPEN guideline on clinical nutrition and hydration in geriatrics. Clin Nutr 38(1):10-47

2. Ministers, NCo (2014) Nordic Nutrition. Recommendations 2012. Integrating nutrition and physical activity

3. Brownie S, Muggleston H, Oliver C (2015) The 2013 Australian dietary guidelines and recommendations for older Australians. Aust Fam Physician 44(5):311-315

4. Age Scotland. Eat Well Age Well Project. (2020) Eat Well: A Guide for Older People in Scotland. https://www.eatwellagewell.org.uk/images/EatWellGuide2020.pdf

5. Shlisky J et al (2017) Nutritional considerations for healthy aging and reduction in age-related chronic disease. Adv Nutr 8(1):17-26

6. Gomes $\mathrm{F}$ et al (2018) ESPEN guidelines on nutritional support for polymorbid internal medicine patients. Clin Nutr 37(1):336-353

7. EFSA Panel on Dietetic Products, NaAENP (2016) Scientific opinion on dietary reference values for vitamin D. EFSA NDA Panel. EFSA J:145

8. Queensland Goverment. Estimating energy, protein \& fluid requirementsfor adult clinical conditions. (2015) Consensus document from Dietitian/Nutritioists from the Nutrition Education Matreal Online "NEMO". Revised May 2015. https://www.scribd.com/document/295124816/ Estimating-Energy-Protein-and-Fluid-Requirements-for-Adult-Clinical-Conditions

9. EatRight Academy of Nutrition and Dietetics. What are the caloric needs of healthy older adults (over age 65)? (2009) Adapted by the Academy of Nutrition and Dietetics from the American Academy of Pediatrics, Classifying Recommendations for Clinical Practice Guidelines, Pediatrics. 2004;114;874-877s. UWL:Energy needs 2009. https://www.andeal. org/recommendation-ratings

10. Dietitian/Nutritionists from the Nutrition Education Materials Online, N., team, Estimating energy, protein \& fluid requirements for adult clinical conditions, p.f.r.f.a.c.c. Estimating energy, Editor. Queensland Government: Queensland Government.

11. O'Keeffe $\mathrm{M}$ et al (2019) Potentially modifiable determinants of malnutrition in older adults: a systematic review. Clin Nutr 38(6):2477-2498

12. Volkert D et al (2019) Development of a model on determinants of malnutrition in aged persons: a MaNuEL project. Gerontol Geriatr Med 5:2333721419858438

13. Cox NJ et al (2019) Assessment and treatment of the anorexia of aging: a systematic review. Nutrients 11(1):144

14. Chang $\mathrm{M}$ et al (2020) A poor appetite or ability to eat and its association with physical function amongst community-dwelling older adults: age, gene/environment susceptibility-Reykjavik study. Eur J Ageing

15. Volkert D, Schrader E (2013) Dietary assessment methods for older persons: what is the best approach? Curr Opin Clin Nutr Metab Care 16(5):534-540 
16. Hengeveld LM et al (2020) Prevalence of protein intake below recommended in communitydwelling older adults: a meta-analysis across cohorts from the PROMISS consortium. J Cachexia Sarcopenia Muscle 11(5):1212-1222

17. Bjornsdottir $\mathrm{R}$ et al (2013) Validation of a plate diagram sheet for estimation of energy and protein intake in hospitalized patients. Clin Nutr 32(5):746-751

18. Worldwide N (2021) NutritionDay worldwide—-benchmark \& monitor your nutrition care. [cited 2021, 31]. https://www.nutritionday.org/

19. Organization WH (2020) Malnutrition-key facts, i. comprehensive implementation plan on maternal, and young child nutrition, adopted by member states through a world health assembly resolution in 2012. WHO, Geneva. https://www.who.int/news-room/fact-sheets/detail/ malnutrition

20. Cederholm T et al (2017) ESPEN guidelines on definitions and terminology of clinical nutrition. Clin Nutr 36(1):49-64

21. Cederholm T et al (2019) GLIM criteria for the diagnosis of malnutrition-a consensus report from the global clinical nutrition community. J Cachexia Sarcopenia Muscle 10(1):207-217

22. Wolters $\mathrm{M}$ et al (2019) Prevalence of malnutrition using harmonized definitions in older adults from different settings—a MaNuEL study. Clin Nutr 38(5):2389-2398

\section{Recommended Reading}

ASPEN Geriatric Care Resources. https://www.nutritioncare.org/Guidelines_and_Clinical_ Resources/Geriatric_Care_Resources/

Canada's Food Guide-Healthy eating for seniors. Date modified 11.12.2019. https://food-guide. canada.ca/en/tips-for-healthy-eating/seniors/

Cederholm Jensen GL et al (2019) GLIM criteria for the diagnosis of malnutrition-a consensus report from the global clinical nutrition community. Clin Nutr 38(1):1-9

National guidelines for geriatric nutrition, for example

Nordic Nutrient Recommendation (2012) Nord 2014:002 (NNR 2012). https://www.norden.org/ en/publication/nordic-nutrition-recommendations-2012

O'Keeffe M et al (2019) Potentially modifiable determinants of malnutrition in older adults: a systematic review. Clin Nutr 38(6):2477-2498

Volkert D, Beck AM, Cederholm T, Cruz-Jentoft A, Goisser S, Hooper L, Kiesswetter E, Maggio M, Raynaud-Simon A, Sieber CC, Sobotka L, van Asselt D, Wirth R, Bischoff SC (2019) ESPEN guideline on clinical nutrition and hydration in geriatrics. Clin Nutr 38(1):10-47

Open Access This chapter is licensed under the terms of the Creative Commons Attribution 4.0 International License (http://creativecommons.org/licenses/by/4.0/), which permits use, sharing, adaptation, distribution and reproduction in any medium or format, as long as you give appropriate credit to the original author(s) and the source, provide a link to the Creative Commons license and indicate if changes were made.

The images or other third party material in this chapter are included in the chapter's Creative Commons license, unless indicated otherwise in a credit line to the material. If material is not included in the chapter's Creative Commons license and your intended use is not permitted by statutory regulation or exceeds the permitted use, you will need to obtain permission directly from the copyright holder. 